\title{
77. Diurnal Rhythms in the Formation of Lamellar Bone in Young Growing Animals
}

\author{
By Hisashi ShinodA and Masahiro OKADA, M. J. A. \\ Department of Experimental Pharmacology, Medical Research Institute, \\ Tokyo Medical and Dental University \\ (Communicated by Takao Fusayama, M. J. A., Dec. 12, 1988)
}

Introduction. Periodic patterns are universally found in the hard tissues of animals such as in bone, teeth and shells, just as there are annual rings in wood.1)-8) The mechanism for the formation of such periodic structures has, however, not been established.

In the late 1930s, Okada first focused on these structural rhythms from the viewpoint of chronobiology. By developing a vital staining method for hard tissues using lead acetate, he demonstrated for the first time that the periodicity of the striation in the rabbit dentin was diurnal, ${ }^{9)}$ and that the formation of the periodic pattern was closely related to various metabolic fluctuations in the animal body such as sleep and wakefulness, metabolic acidosis and alkalosis, or drug actions. ${ }^{1)}$

In view of the diurnal layering found in dentin, a similar layering would be expected in bone. However, very little investigation along these lines have been conducted on bone, except for the first observation by Okada and Mimura on the diurnal lamellar bone formation, ${ }^{8}$ probably due to two reasons. First, the structure in bone reflects repetitive formation and resorption and therefore is relatively more complicated. Secondly, compared to dentin, the lamellar structure in bone is more difficult to observe because the periodic change is dependent on collagen fiber arrangement rather than the composition of the matrix or the mineral density as in dentin.

Using phase-contrast microscopy, for much clearer observations than previously, and by utilizing a slight modification of the above vital staining method, chronologic studies of lamination processes in all of the four types of lamellar bone were made.

Materials and methods. 1) Experimental animals. The experiment started with 24 male Wistar rats with a mean body weight of $124 \mathrm{~g}, 8$ male Asiatic chipmunks (Tamias sibiricus) with a mean body weight of $62 \mathrm{~g}, 6$ male Japanese white rabbits with a mean body weight of $860 \mathrm{~g}$ and a male Beagle dog with a body weight of $11.3 \mathrm{~kg}$. The animals were adapted for $2 \mathrm{wks}$ to an environmental room equipped with an automatically timed $12 \mathrm{~h} / 12 \mathrm{~h}$ light-dark illumination program (light on from 6:00 to $18: 00$ ) at a temperature of $24 \pm 1{ }^{\circ} \mathrm{C}$ and a relative humidity of $55 \pm 3 \%$. They were fed standard laboratory chow (MF-2 for rats and chipmunks, GC-4 for rabbits, and DS for dog, Oriental Yeast Co., Ltd., Tokyo) and deionized water ad libitum.

2) Observation of periodicity in the formation of lamellar bone. In order to observe the periodicity in the lamellar bone formation, a vital staining method with lead salts originally developed by Okada and Mimura, ${ }^{9)}$ and recently modified by Asoda et al.10) for subcutaneous injection was applied. The lead labeled line $(\mathrm{Pb}$ line) can clearly indicate the time of formation since it appears at the site 
of ongoing calcification within about $30 \mathrm{sec}$ following the injection of $\mathrm{Pb}$ salts. The $\mathrm{Pb}$ mark is not lost by decalcification of specimens in contrast to the techniques using tetracyclines or other markers which are readily lost by decalcification. The vital staining was performed as follows: A small amount of nitrilotriacetato lead (NTA-Pb, $0.3-0.5 \mathrm{mg} \mathrm{Pb} / 100 \mathrm{~g}$ body weight) was subcutaneously injected into the animal. The injection was repeated at intervals of 2 to 11 days. After sacrificing the animal with a lethal dose of ether or pentobarbital, hard tissues including femur, tibia and mandibula were taken and fixed in Karnovski's solution. The tissues were next decalcified for 5-7 days in $0.2 \mathrm{~N}$ $\mathrm{HCl}$ saturated with $\mathrm{H}_{2} \mathrm{~S}$ gas to fix the $\mathrm{Pb}$ in the matrix as insoluble $\mathrm{PbS}$. The decalcified tissues were embedded in gelatin and frozen sections of $6-8 \mu \mathrm{m}$ were prepared using a cryostat. The sections were then immersed in $0.1 \% \mathrm{HAuCl}_{4}$ to gild the brownish $\mathrm{Pb}$ lines until the desired intensity and color (purplish black) appeared. The sections were next fixed in $5 \% \quad \mathrm{Na}_{2} \mathrm{~S}_{2} \mathrm{O}_{3}$ and washed with water. All of the sections were mounted in glycerin jelly and observed using a phasecontrast microscope (Axiophoto, Carl Zeiss, West Germany). Chronologic analyses of lamellar bone formation were performed by counting the number of lamellae between the $\mathrm{Pb}$ lines.

3) Types of bone examined. Four types of bone were observed: the endosteum (inner circumferential lamella) and the periosteum (outer circumferential lamella) of non-Haversian bone, and the primary and secondary lamellae of Haversian bone. The portion of bone with significantly vigorous one-way growth was selected for the observation.

Results. The number of lamellae that could be counted between the $2 \mathrm{~Pb}$ labeled lines was generally correspondent to the number of days between the NTA-Pb injections. Representative examples are shown in the following figures.

Fig. 1 shows the inner circumferential lamella in a cross section of rat femur diaphysis. In this case, NTA-Pb was injected twice at an 11-day interval. Eleven lamellae were counted between the $2 \mathrm{~Pb}$ lines.

Fig. 2 shows the outer circumferential lamella in a cross section of femur diaphysis in an Asiatic chipmunk. NTA-Pb was injected 5 times at 7 dayintervals. In each area between 2 neighbouring $\mathrm{Pb}$ lines, 7 malellae were observed.

Fig. 3 shows the primary Haversian lamella in a cross section of mandibula in a rabbit. Seven striations were seen between the $2 \mathrm{~Pb}$ lines injected at a 7 day-interval.

Fig. 4 shows the secondary Harversian lamella in a cortical bone of femur diaphysis in a dog. NTA-Pb was injected 4 times at 10 day-intervals. In the area between the 1st and 2nd, and the 2nd and $3 \mathrm{rd} \mathrm{Pb}$ lines, 10 lamellae were observed. In this figure, however, only 5 lamellae could be counted between the 3rd and 4 th $\mathrm{Pb}$ lines although the injections were made at a 10 day-interval. For the Haversian lamellae in the dog, the number of lamellae between $2 \mathrm{~Pb}$ lines were often less than the number of days between injections. However, the number of lamellae never exceeded the number of days between the injections.

Discussions. The number of lamellae correspondent to the number of days between injections was always found in bone where growth was vigorous. This indicates that the lamellar bone is formed by the functioning of osteoblasts in a diurnal rhythm.

In bone where growth was not vigorous, however, the number of lamellae between the 2 labeled lines was found to be less than the number of days between injections (Fig. 4). The osteoblastic function appears to sometimes stop or rest. 

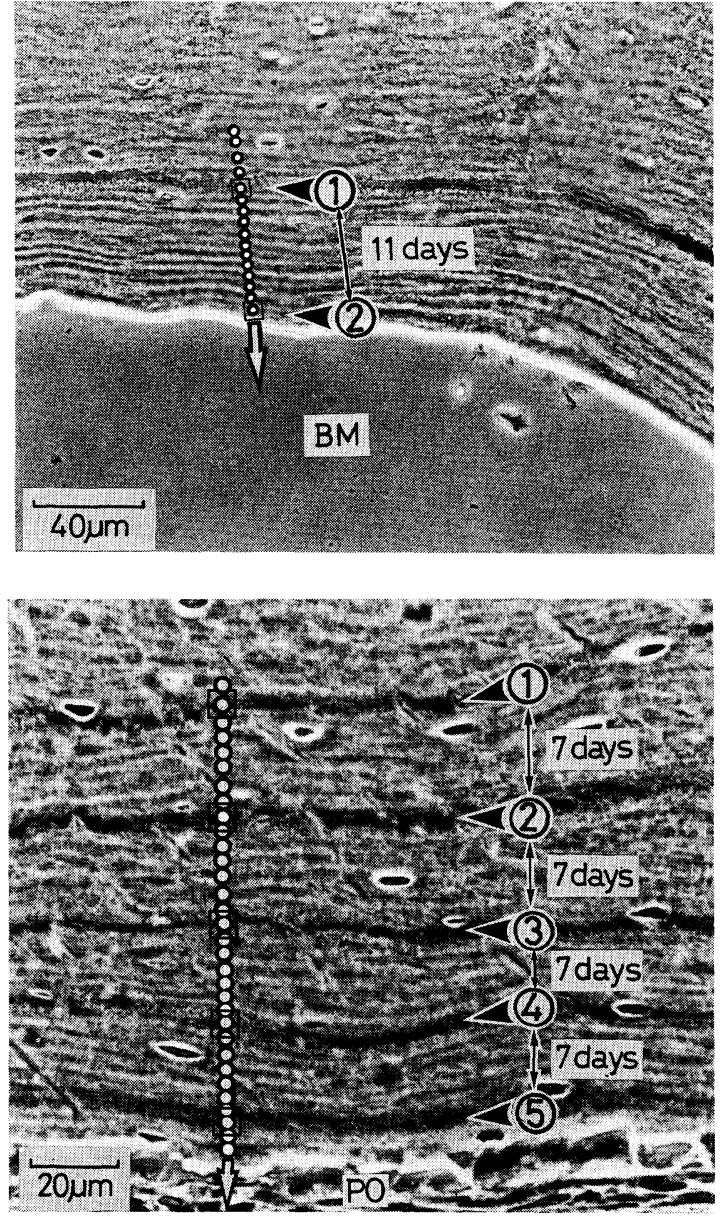

Fig. 1. Inner circumferential lamella in rat femur. (1), (2): $\mathrm{Pb}$ labeled lines. White arrow: Direction of bone formation. $\mathrm{BM}$ : Bone marrow.
Fig. 2. Outer circumferential lamella in femur of Asiatic chipmunk. (1)-(5): $\mathrm{Pb}$ labeled lines. White arrow: Direction of bone formation. PO: Periosteum.

This phenomenon was often observed with the Haversian lamella where the Haversian canal was filled up and consequently had less space to grow. Aging of osteoblasts may also be a factor because a decreased number of lamellae was also observed in non-Haversian bones.

There is general agreement that bone lamella contains fine collagen fibers which run parallel in a lamella but altering the direction by about $90^{\circ}$ in the next lamella. ${ }^{3)}$ The arrangement of collagen fiber seems to undergo alteration during the day and the night. If the movement of osteoblasts affects fiber orientation, lamellar bone presumably reflects a regular diurnal track of cell movement. Unfortunately, the nature and the factors which regulate and instruct the osteoblasts have yet to be clarified.

Our present findings establish the existence of a diurnal periodicity in lamellar bone formation in the growing bone of animals, and together with previous observations in teeth, bone and shells, ${ }^{1), 4)-9}$ ) suggest that the hard tissues of animals generally grow in a diurnal rhythm. The rhythmic activity of hard tissue forming cells can thus be recorded on the hard tissues. Since the function of tissue cells is invariably under the control of the central nervous system or hormonal effects, our research technique using time-marked hard tissue 
[Vol. 64(B),
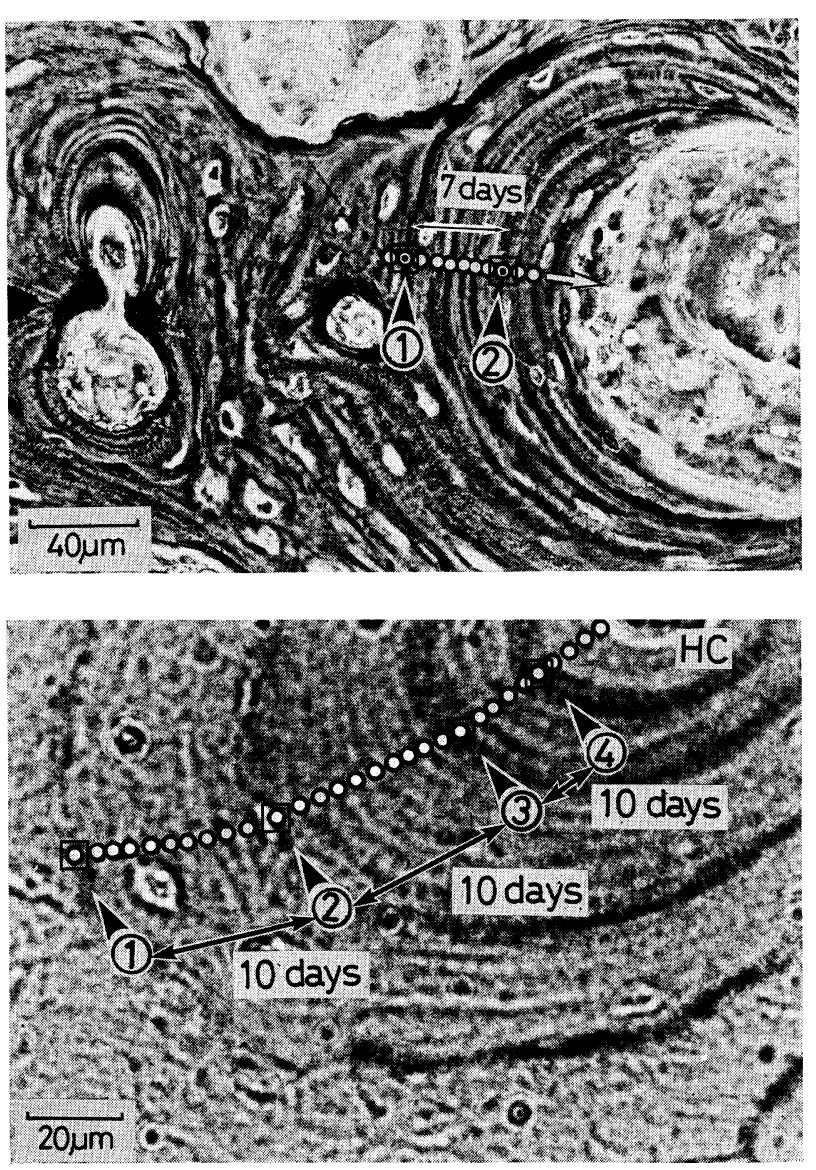

Fig. 3. Primary Haversial lamella in Bucco-lingual cross section of rabbit mandibula. (1), (2): $\mathrm{Pb}$ labeled lines. White arrow: Direction of bone formation.
Fig. 4. Secondary Haversian lamella in dog femur. (1)(4): $\mathrm{Pb}$ labeled lines. $\mathrm{HC}$ : Haversian canal.

as a "kymograph" would be able to provide a method for future studies to clarify or elucidate various such factors that relate to activity or reaction in animals.

Acknowledgments. The authors are indebted to Prof. A. Asoda for continuing encouragement, to Mr. T. Sano for technical assistance and to Prof. T. Fusayama, M. J. A., for reviewing the manuscript.

\section{References}

1) Okada, M. (1943) : Shanghai Evening Post (Sept.), pp. 26-31.

2) Schour, I., and Steadman, S. R. (1935): Anat. Rec., 63, 325-333.

3) Hancox, N. M. (1972): Biology of Bone. Cambridge Univ. Press, London, New York, pp. 24-35.

4) Shinoda, H., and Ogura, H. (1977): Jap. J. Oral Biol., 19, 481-489.

5) Yilmaz, S. et al. (1977): Arch. Oral Biol., 22, 511-513.

6) Kawasaki, K. et al. (1980): ibid., 24, 939-943.

7) Rosenberg, G. D., and Simmons, D. J. (1980) : Calcif. Tissue Int., 32, 29-44.

8) Okada, M., and Mimura, T. (1940): J. Med. Sci. IV., Pharmacology Vol. XIII, nos. $1 / 2,95^{*}-97 *$.

9) - (1938) : ibid., Pharmacology Vol. XI, no. 2, 166*-170*.

10) Asoda, A. et al. (1982) : Annual Report of Med. Res. Inst., Tokyo Med. Dent. Univ., 10, 87-89. 